\title{
Radiographic Features in a Patient Diagnosed with Neurosyphilis
}

\author{
Yoon Ah Park ${ }^{*}$ (D), Jung Woo Ann ${ }^{\dagger}$ (D), Seok Hwi Jeon ${ }^{\dagger}$ (D), Eun Joo Chung ${ }^{\dagger, \ddagger}$ (D), Eung Gyu $\operatorname{Kim}^{\dagger}$ (D),

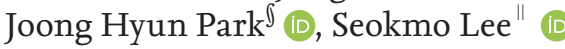 \\ Department of Neurology, Kosin University College of Medicine ${ }^{\star}$, Busan; Department of Neurology, Busan Paik Hospital, Inje University College \\ of Medicine ${ }^{\dagger}$, Busan; Dementia and Neurodegenerative Disease Research Center, Inje University ${ }^{\ddagger}$, Gimhae; Department of Neurology, Sanggye \\ Paik Hospital, Inje University College of Medicine ${ }^{\complement}$, Seoul; Department of Nuclear Medicine, Busan Paik Hospital, Inje University College of \\ Medicine", Busan, Korea
}

The clinical features and brain magnetic resonance image (B-MRI) findings in neurosyphilis (NS) are highly varied. Cortical atrophy, especially involving the medial temporal lobes (MTL), is a remarkable radiographic feature, reported in B-MRI scans, in cases of NS presenting with severe cognitive impairments. The observed MTL atrophy is similar to the atrophy seen in neurodegenerative disorders such as Alzheimer's disease (AD) or frontotemporal dementia (FTD). A fluorodeoxy-glucose-positron emission tomography (FDG-PET), used for the diagnosis of AD, commonly reveals global cortical hypometabolism, sparing basal ganglia and thalamus, in NS. We report a case of NS, with complex symptoms and bilateral MTL atrophy. FDG-PET imaging showed bilateral thalamic and right visual cortical glucose reduction, as well as diffuse cortical hypometabolism. This type of FDG-PET pattern is a new finding in the field of NS.

J Neurosonol Neuroimag 2019;11(2):173-177

Key Words: Neurosyphilis; Magnetic resonance imaging; Mesial temporal lobes; Fluorodeoxyglucose F18 positron-emission tomography
Received: November 4, 2019

Revised: December 2, 2019

Accepted: December 13, 2019

Address for correspondence: Eun Joo Chung

Department of Neurology, Busan Paik Hospital, Inje University College of Medicine, 75 Bokji-ro, Busanjin-gu, Busan 47392, Korea

Tel: $+82-51-890-6409$

Fax: $+82-51-895-6367$

E-mail: strokerchung@gmail. com
Neurosyphilis (NS) is an infection of the central nervous system, caused by the invasion of Treponema pallidum, which can occur in any stage of syphilis, particularly in the late stages of the disease. ${ }^{1}$ NS presents with various clinical features, such as mimicking early onset Alzheimer's disease (AD), mild frontotemporal dementia (FTD) or primary psychiatric disease (PPD). ${ }^{1-3}$ Brain magnetic resonance imaging (B-MRI) features seen in NS vary, and are usually presented as cerebral infarction, sulci widening, global/hippocampal predominant atrophy, white matter lesions, or meningitis. ${ }^{4-7}$ Recently, diffuse temporoparietal hypometabolism and spared thalamic metabolism, in fluorodeoxy-glucose-positron emission tomography (FDG-PET), have been reported as new findings in NS. ${ }^{3}$

In the current case report, the authors describe a patient with NS, who exhibited varied and complex clinical symptoms. B-MRI showed severe atrophy, involving bilateral medial temporal lobes (MTL), and abnormalities were detected in FDG-PET scan.

\section{CASE REPORT}

A 49-year-old male patient was hospitalized with a history of psychiatric manifestations since 10 months, personality changes, and progressive cognitive impairments. The patient had a history of exhibiting depressive behavior and at times anger, for about 10 months before admission. He also displayed the habit of repeating words and frequently mentioning past episodes. Sometimes, during conversations, the patient interrupted inappropriately or spoke on irrelevant subjects.

Approximately 7-month before hospitalization, the patient experienced delusion and insomnia. He believed he was a genius with an IQ of 190. Occasionally, 
he exclaimed that someone in the mirror was looking at him. When he went out alone, he could not find his way back home and a police officer had to accompany him back. Approximately one month before hospitalization, he had recurrent episodes of falling and exhibited features of dysarthria. He did not recognize himself in the mirror and misidentified himself as someone else. As time progressed, he became more aggressive and displayed poor personal hygiene and urinary incontinence.

No relevant medical, social, or family histories were noted. The patient was a right-handed high school graduate.

Mini-mental state examination score was 20/30 (orientation in time $2 / 5$; orientation in place $5 / 5$, memory registration 3/3; serial 7 ' score 2/5; delayed recall 1/3, language related $7 / 8$; pentagon copy $\mathrm{o} / 1$ ). Clinical dementia rating (CDR) scale was 1 and CDR-sum of box score was 7. Korean instrumental activities of daily living score was 1.1. Neuropsychological assessment showed abnormal findings in all cognitive domains including visual/ verbal, memory, language, visuospatial, and frontal executive functions. Particularly, the patient's memory impairment was found to be a storage dysfunction. Psychiatric inventory revealed agitation, apathy, irritability, and disinhibition. No physical or neurological abnormalities were detected.

Blood tests related to young onset dementia: serum rapid plasma reagin and the fluorescent treponemal antibody absorption tests (immunoglobulin G and immunoglobulin M) were observed to be reactive. Serum treponema pallidum hemagglutination assay (TPHA) was found to be positive (1:320).

B-MRI showed bilateral temporal lobe atrophy, involving the hippocampus, and hyperintensity in the splenium and both occipital horns of lateral ventricles (Fig. 1). No abnormalities were detected on diffusion-weighted image, gadolinium-enhanced T1 weighted images, and magnetic resonance angiography.

FDG-PET showed severe decline in glucose metabolism in bilateral frontal, parietal, and temporal lobes including the hippocampus (more prominently decreased in the right hemisphere), and both thalami (Fig. 2).

Cerebrospinal fluid (CSF)-white blood cell count was $O$ and the ratio of CSF glucose to serum glucose was normal. CSF-protein was elevated $(85.9 \mathrm{mg} / \mathrm{dL})$ and CSF-venereal disease research laboratory was reactive. CSF-TPHA was positive (titer, 1:1280). There was no evidence of other infections (fungal, bacterial, tuberculous, viral) or malignant cytology in the CSF analysis.

Despite being diagnosed with NS, the patient refused treatment. In the one and a half months following discharge, the patient's behavioral and psychological symptoms worsened and he was subsequently admitted to a long-term care facility.

\section{DISCUSSION}

Diffuse cortical atrophy is one of the major findings in NS. ${ }^{1,4,6,7}$ In particular, atrophy of the bilateral medial temporal lobes (MTL) and hippocampus are more pronounced. ${ }^{1-3,5}$ If patients with NS show bilateral MTL atrophy and cognitive impairments like $\mathrm{AD}$, they might
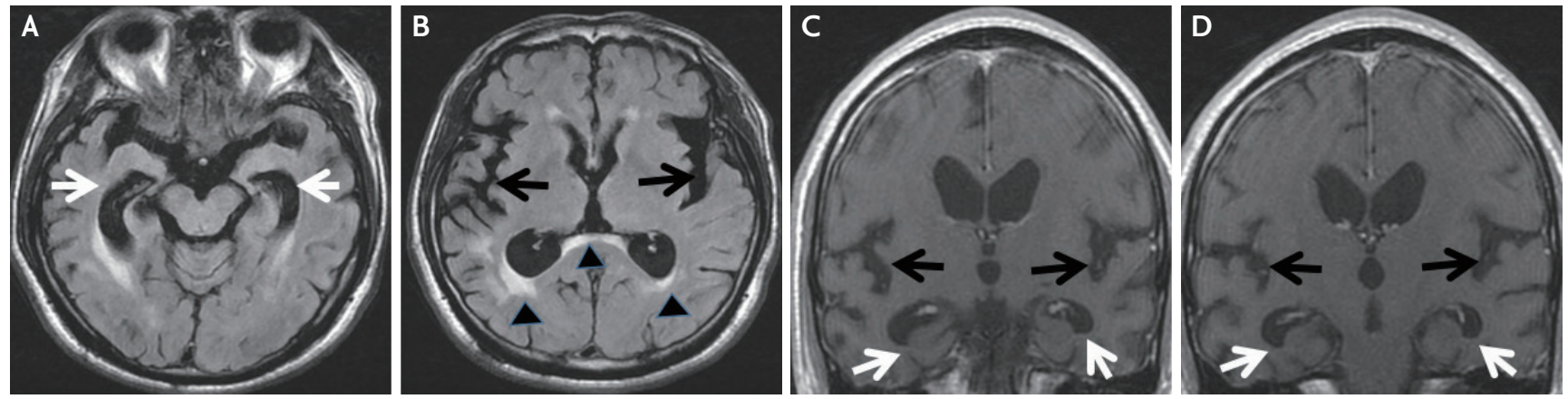

FIG. 1. (A, B) Axial FLAIR magnetic resonance images showing atrophy in bilateral medial temporal lobes (white arrows of A), widening of both sylvian fissures (black arrows of B), and high signal intensities in the splenium and in both occipital horns of lateral ventricles (black arrowheads of $\mathrm{B}$; more severe in the right occipital horn). (C, D) Coronal T1-weighted image showing atrophy in the bilateral medial temporal lobes (white arrows) and widening of both sylvian fissures (black arrows). 

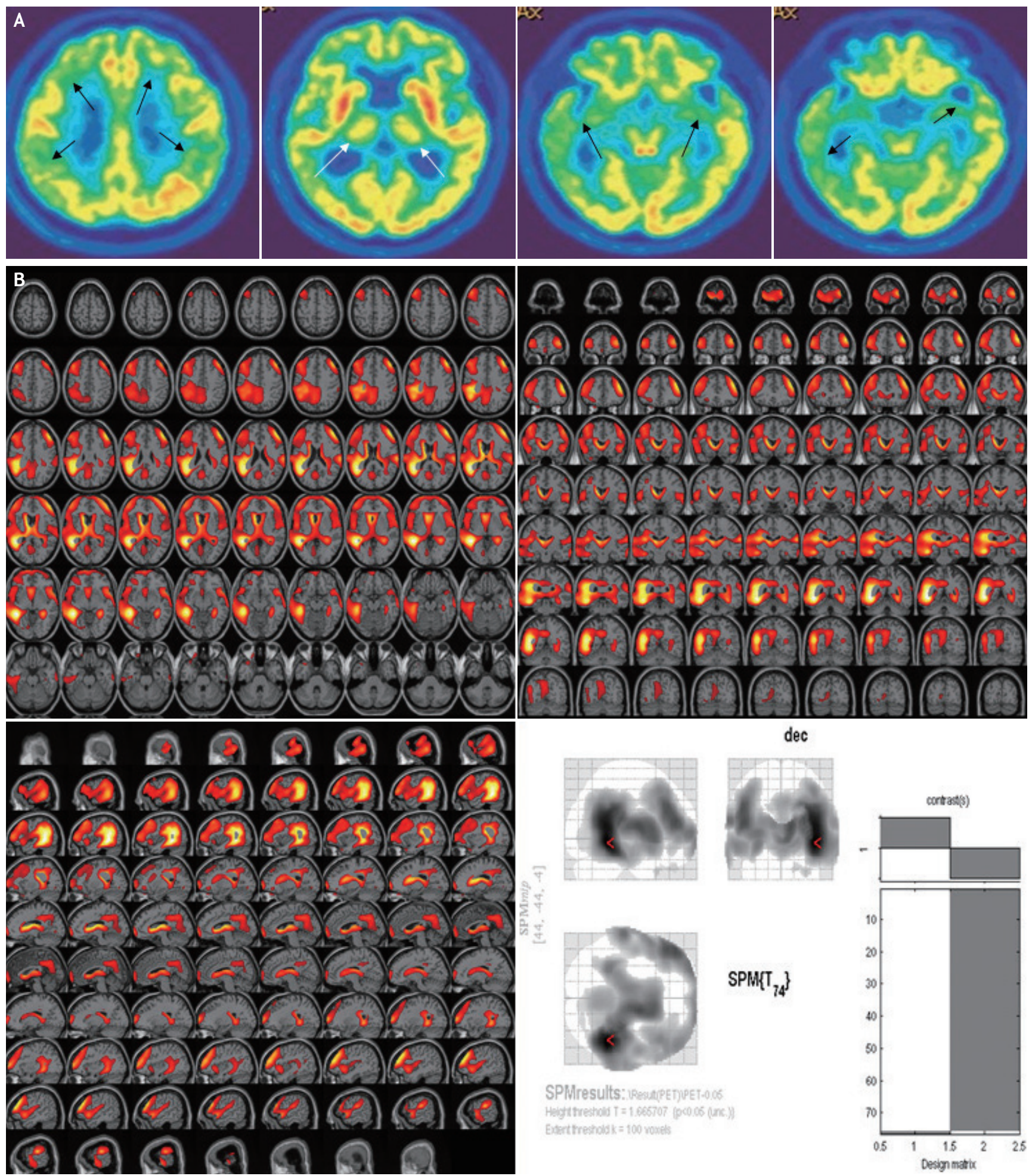

FIG. 2. Fluorodeoxy-glucose-positron emission tomography. (A) Hypometabolism observed in bilateral frontal, parietal, and temporal lobes (more prominent on the right) (black arrows), and thalamus (white arrows). (B) Statistical parametric mapping analysis showing the hypometabolism in bilateral frontal, parietal, and temporal lobes, compared with age-matched controls. 
be misdiagnosed as cases mimicking $\mathrm{AD}^{2,3}$ However, if patients show severe atrophy and high signal changes in the MTL with psychiatric symptoms, these can be confused with the typical radiologic manifestations of viral or limbic encephalitis. ${ }^{5}$ In addition, clinical symptoms of NS are, at times, confused with FTD or PPD., ${ }^{1,2}$ Therefore, specific clinical and B-MRI findings associated with NS can often help avoid the confusion in diagnosis.

This patient in this case, presented complex clinical symptoms such as personality change, psychiatric manifestations, and cognitive impairments. His personality change and psychiatric symptoms could be confused with FTD. The patient showed poor results in all cognitive domains. In addition, the patient's memory function could not be recovered with hints thus exhibiting a storage impairment pattern. Previous literature supports this observation through a case report, ${ }^{2}$ in which memory impairment in NS was observed to have a pattern similar to that of AD. Memory dysfunction in our patient was also associated with severe atrophy in MTL.

FDG-PET findings in NS have been highly varied,.$^{3,8} \mathrm{~A}$ previous study showed diffuse hypometabolism in the frontal areas with preserved glucose consumption in basal ganglia and thalamus, in NS. ${ }^{8}$ Recently, FDG-PET findings with severe hypometabolism of diffuse temporal cortex, orbitofrontal lobe, and the precuneus/posterior cingulate without abnormal thalamic metabolism have been reported in NS. ${ }^{3}$ Diffuse cortical hypometabolism in our patient is similar to the aforementioned reports. However, our patient showed thalamic hypometabolism in FDG-PET imaging. Hypometabolism in the parietotemporal association cortices, and the frontal cortices, have been commonly observed in patients with early $\mathrm{AD}$, whereas, the metabolism in the thalamus is relatively preserved. ${ }^{3}$

Cognitive dysfunctions in our patient are similar to the features seen in $\mathrm{AD}$, despite the clinical features mimicking FT'D and/or PPD. B-MRI findings also revealed MTL atrophy and diffuse cortical atrophy mimicking $\mathrm{AD}$. In such clinical situations, serologic tests and lumbar punctures should be considered due to the difficulty in diagnosing NS. Cognitive decline, in our patient, is most probably due to the MTL atrophy and cortical hypometabolism (frontal, parietal, and temporal cortex).
Another report suggests the differences in FDG-PET findings between $\mathrm{AD}$ and NS as follows: high T2 signal intensity and atrophy in the bilateral mesial temporal areas are correlated with intensely increased FDG uptake of the hippocampus. ${ }^{9}$ Therefore, FDG-PET is probably helpful in differentiating NS from other diseases with complex symptoms and MTL atrophy. The disturbances in cortical-thalamic-cortical circuitry, due to bilateral thalamic hypometabolism, are probably associated with the delusion and psychiatric symptoms in the patient in this case.

Although an improvement in hypometabolism, after NS treatment, has been reported in previous literature, our report has a limitation in that aspect, as the patient in this case did not undergo treatment and thus, the post-treatment FDG-PET changes could not be assessed.

\section{Conflicts of Interest}

No potential conflicts of interest relevant to this article was reported.

\section{REFERENCES}

1. Yanhua W, Haishan S, Le H, Xiaomei Z, Xinru C, Ling L, et al. Clinical and neuropsychological characteristics of general paresis misdiagnosed as primary psychiatric disease. BMC Psychiatry. 2016;16:230.

2. Wang J, Guo Q, Zhou P, Zhang J, Zhao Q, Hong Z. Cognitive impairment in mild general paresis of the insane: $\mathrm{AD}$-like pattern. Dement Geriatr Cogn Disord. 2011;31:284-290.

3. Verjans S, Van Laere K, Vandenberghe R. Neurosyphilis mimicking young-onset Alzheimer's disease: a case report explaining the pitfalls of FDG-PET. Acta Neurol Belg. 2016;116:207-210.

4. Peng F, Hu X, Zhong X, Wei Q, Jiang Y, Bao J, et al. CT and MR findings in HIV-negative neurosyphilis. Eur J Radiol. 2008;66:1-6.

5. Jeong YM, Hwang HY, Kim HS. MRI of neurosyphilis presenting as mesiotemporal abnormalities: a case report. Korean J Radiol. 2009;10:310-312.

6. Khamaysi Z, Bergman R, Telman G, Goldsher D. Clinical and imaging findings in patients with neurosyphilis: a study of a cohort and review of the literature. Int J Dermatol. 2014;53:812-819. 
7. Włodarczyk A, Szarmach J, Jakuszkowiak-Wojten K, GałuszkoWęgielnik M, Wiglusz MS. Neurosyphilis presenting with cognitive deficits - a report of two cases. Psychiatr Danub. 2017;29:341-344.

8. Pichler R, Doppler S, Szalay E, Hertl C, Knell U, Winkler J. SPECT and FDG-PET in diagnostics of neurolues. Wien
Klin Wochenschr 2008;120:20-23.

9. Omer TA, Fitzgerald DE, Sheehy N, Doherty CP. Neurosyphilis presenting with unusual hippocampal abnormalities on magnetic resonance imaging and positron emission tomography scans: a case report. J Med Case Rep. 2012;6:389. 